\title{
La sécrétion acide de l'estomac: un mécanisme biologique peu commun
}

Miguel J.M. Lewin

Directeur de l'Unité de recherches de gastroentérologie de l'Inserm (UIO).

\section{REFERENCES}

1. Ito S. Functional gastric morphology. In: Johnson LR, ed. Physiology of the Gastrointestinal Tract. New York : Raven Press, 1981 : 517-50.

2. Mignon M, Galmiche JP, Lewin MJM. Physiologie de l'Estomac. In: Meyer P, éd. Physiologie Humaine ( $2^{\mathrm{e}}$ édition). Paris : Flammarion Médecine Sciences, $1983: 190-223$.

3. Lewin MJM. Hormone receptor control of electrolyte secretion in the gastrointestinal tract. In: Glass GBJ, ed. Gastrointestinal Hormones. New York : Raven Press, 1980 : 529-64.

4. Black JW, Duncan WAM, Durant CJ, Ganellin CR, Parsons EM. Definition and antagonism of histamine H2-receptors. Nature 1972; $236: 385-90$.

5. Cheret AM, Pignal F, Lewin M JM. Eff ects of $\mathrm{H} 2$-receptor antagonists cimetidine, ranitidine, and ICI 125,211 on histamine-stimulated adenylate cyclase activity in guinea pig gastric mucosa. Mol Pharmacol 1982; 20 : 326-30.

\section{ADRESSE}

MJM Lewin : Inserm U10, hôpital Bichat, 170 , boulevard Ney, 75877 Paris cedex 18

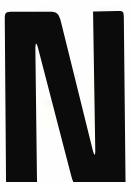

otre estomac sćcrète chaque jour deux à trois litres d'une solution concentrée d'acide chlorhydrique. Cette sécrétion "acide" est le fait de cellules appelées "pariétales" ou "bordantes" ou encore "oxyntiques", situées principalement au niveau du collet des tubes glandulaires de l'épithélium fundique, dans la partie supérieure de l'estomac (figure $t$ ). Elle est accompagnée d'autres sécrétions quantitativement moins importantes, provenant soit des cellules pariétales, soit des autres cellules exocrines de l'estomac : du bicarbonate de sodium et des glycoprotéines sécrétés par les cellules à mucus tapissant la surface de tout l'estomac ainsi que le haut des tubes glandulaires antraux et fundiques, du pepsinogène sécrété par les cellules "principales" localisées au fond des tubes glandulaires fundiques et le "facteur intrinsèque " sécrété par les cellules pariétales [1].

\section{Importance de l'ion $\mathrm{H}^{+}$}

Mis à part la sécrétion du facteur intrinsèque, indispensable à l'absorption de la vitamine $B_{12}$, c'est la sécrétion acide qui joue le plus grand rôle dans la phase gastrique de la digestion. Par son volume, elle dilue les aliments et participe à la régulation de leur temps de transit dans l'estomac. Par son acidité, elle exerce une action bactériostatique, elle active le pepsinogène en pepsine qui amorce la digestion des protéines, elle freine, au niveau de l'antre gastrique, la libération de la gastrine, hormone , stimulant la sécrétion acide et elle déclenche, au niveau du duodénum, la libération de sécrétine, hormone stimulant la sécrétion pancréatique externe. La sécrétion gastrique acide joue aussi un rôle très important dans le mécanisme de l'ulcérogenèse. On ignore, à ce jour, la nature première et l'enchaînement des processus aboutissant à la formation de la lésion ulcéreuse, mais on sait que la neutralisation ou l'inhibition de la sécrétion acide par des agents pharmacologiques ou antisécrétoires permet dans la plupart des cas sa guérison (voir l'article de S. Bonfils p. 24I-7).

\section{Une régulation physiologique complexe}

L'activation de la cellule pariétale est le résultat de l'intégration de plusieurs facteurs hormonaux et nerveux agissant localement ou à distance et faisant intervenir de nombreuses boucles de rétrocontrôle (figure 2, voir p. suivante) [2].

Pour répondre à tous ces stimuli, la cellule pariétale est équipée de plusieurs systèmes "récepteurs " localisés sur la face basolatérale (côté 
"milieu intérieur "), ayant pour fonction de reconnaître spécifiquement les divers médiateurs et de transmettre leur information à l'intérieur de la cellule [3]. La spécificité de reconnaissance entre un médiateur donné et son récepteur est basée sur une complémentarité de structure chimique qui se traduit par une affinité réciproque. La

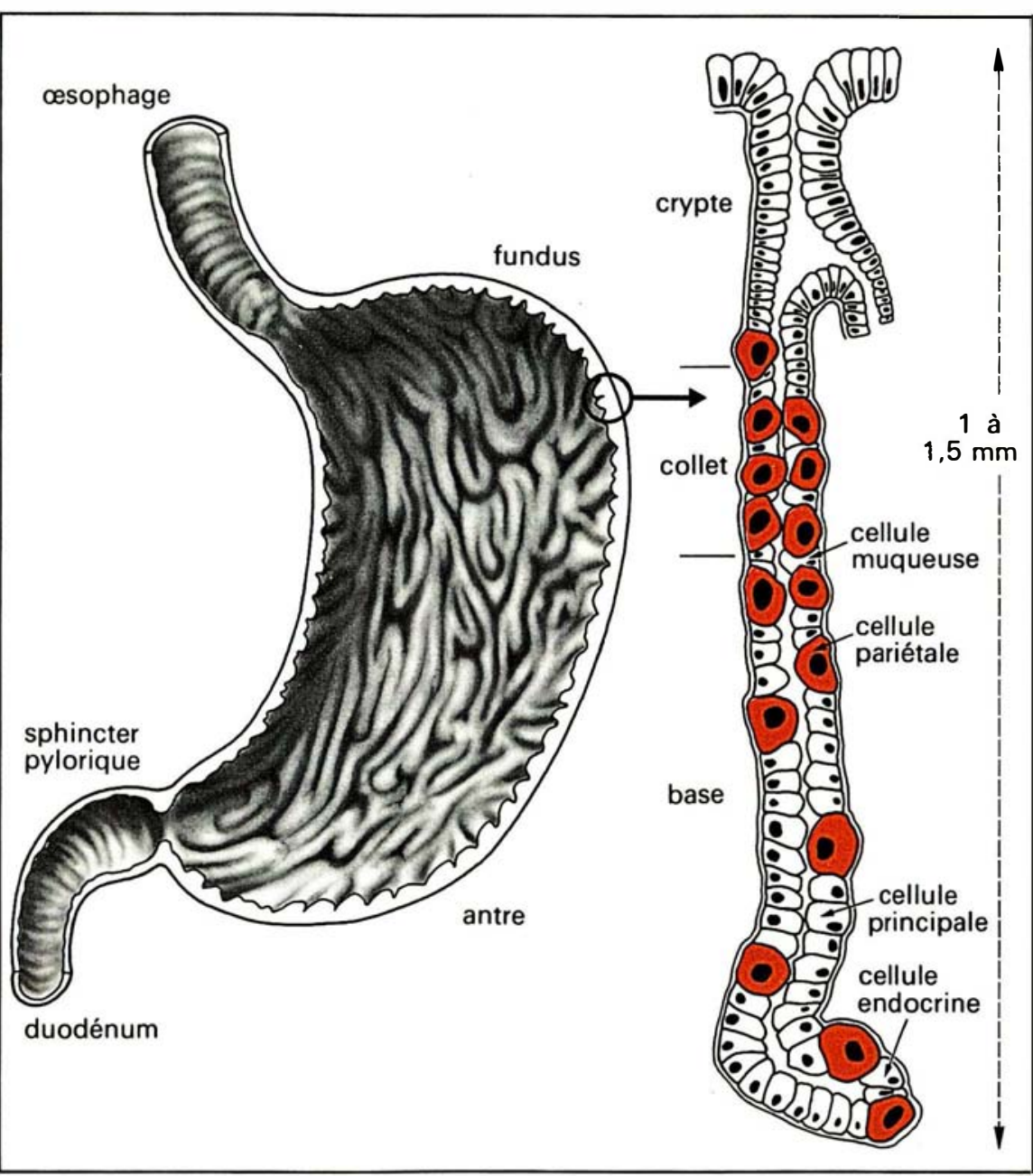

Figure I. Localisation de la cellule pariétale (acido-sécrétrice) dans l'estomac. La coupe, à gauche, montre la muqueuse gastrique, épithélium plissé s'étendant entre l'oesophage et le sphincter pylorique qui sépare l'estomac du duodénum. On distingue deux principales zones: le fundus, dans la partie supérieure de l'estomac, l'antre dans la partie inférieure. Le fundus est la partie exocrine proprement dite alors que l'antre contient l'essentiel des cellules endocrines gastriques.

L'épithélium fundique est constitué de tubes glandulaires dont un schéma est représenté à droite. Ce tube comprend trois parties principales : la crypte tapissée de cellules à mucus, le collet constitué de cellules muqueuses et de cellules pariétales, la base contenant quelques cellules pariétales, les cellules principales (sécrétant le pepsinogène) et de rares cellules endocrines (parmi lesquelles des cellules à somatostatine).

$m / s n^{\circ} 5$ septembre 85 connu. Il n'est pas semblable au récepteur histaminique du muscle lisse (type " $\left.\mathrm{H}_{1} »\right)$ mais fait partie de la famille des récepteurs " $\mathrm{H}_{2}$ ". Ces récepteurs, qui sont également présents au niveau du cœur, de l'utérus et du cerveau sont caractérisés par une classe d'antagonistes spécifiques $\lceil 4\rceil$ dont font partie la cimétidine (ou Tagamet $\mathbb{B}$ ) et la

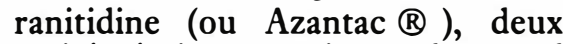
antisécrétoires gastriques de grand intérêt thérapeutique [5]. Le récepteur $\mathrm{H}_{2}$ est couplé à une adénylate cyclase par l'intermédiaire de sousunités de régulation, elles-mêmes contrôlées par le guanine nucléotide triphosphate (GTP). La liaison de l'histamine au récepteur active l'adénylate cyclase conduisant à la formation d'AMP cyclique. Ce second messager active à son tour une ou plusieurs protéines kinases qui vont enfin stimuler le transport de $\mathrm{HCl}$ en catalysant la phosphorylation de certaines protéines. Grâce à ce récepteur, l'histamine joue un rôle de premier plan dans la stimulation de la cellule pariétale. On ignore cependant son origine cellulaire exacte : elle pourrait être sécrétée par des cellules épithéliales spécialisées ou par des fibres nerveuses " histaminergiques".

Le récepteur cholinergique est de type muscarinique. Il agirait en déclenchant, au niveau de la membrane basolatérale, l'ouverture d'un canal permettant l'entrée d'ions $\mathrm{Ca}^{2+}$ dans la cellule [6]. Cette ouverture pourrait impliquer un second messager particulier, l'inositol triphosphate, résultant lui-même du clivage des phosphoinositides membranaires par une phosphodiestérase spécifique. L'augmentation intracellulaire de $\mathrm{Ca}^{2+}$ provoquerait la stimulation de protéines kinases couplées à des calci-protéines de type calmoduline, le résultat étant, comme pour l'AMP cyclique, l'activation du transport de $\mathrm{HCl}$.

Le récepteur cholinergique de la cellule pariétale ne paraît pas présenter de caractéristiques structurales particulières, mais un récepteur " $M_{1}$ " spécifiquement antagonisé par un certain type de molécule (la pirenzepine) pourrait être présent, en amont de cette cellule, sur le trajet de l'innervation vagale [7].

Le récepteur gastríinique n'a pas 


\section{RÉFÉRENCES}

6. Lewin MJM, Sachs G. Mécanismes cellulaires et moléculaires de la sécrétion gastrique acide. In: Lewin MJM, Mignon M, ed., La Muqueuse Gastrique et ses Sécrétions. Paris: Smith Kline \& French, $1985: 17-33$

7. Hirschowitz BI, Hammer R, Giachetti A, Keirns JJ, Levine R. Subtypes of Muscarinic Receptors. Trends in Pharmacological Sciences 1983 , supplement.

8. Soumarmon A, Cheret AM, Lewin MJM. Localization of gastrin receptors in intact isolated and separated rat fundic cells. Gastroenterology 1977; $73: 900-3$.

9. Soll AH, Amirian DA, Thomas LP et al. Gastrin receptors on nonparietal cells isolated from canine fundic mucosa. Am 7 Physiol 1984; $247: G_{715-23}$.

10. Jakobs KH, Aktories K, Schultz G. A nucleotide regulatory site for somatostatin inhibition of adenylate cyclase in $\mathrm{S}_{49}$ lymphoma cells. Nature $1983 ; 303: 177-8$.

11. Reyl F, Lewin MJM. Intracellular receptor for somatostatin in gastric mucosal cells: decomposition and reconstitution of somatostatinstimulated phosphoprotein phosphatases. Proc Natl Acad Sci USA 1982; $79: 978-82$.

12. Lewin MJM. Somatostatin receptors. Scand 7 Gastroenterol 1985 (in press).

13. Lewin MJM. Anatomie et physiologie cellulaire: mécanisme et régulation de la sécrétion acide gastrique à l'échelon cellulaire. In: Bernier JJ, éd. Traité de Gastroentérologie. Paris : Flammarion, $1984: 226-30$.

14. Ganser AL, Forte JG. Ionophoretic stimulation of $\mathrm{K}^{+}$-ATPase of oxyntic cell microsomes. Biochem Biophys Res Commun 1973; 54 : 690-6.

15. Hollemans M, Donker-Koopman W, Tager JM. A critical examination of the evidence for an MgATP-dependent proton pump in rat liver lysosomes. Biochim Biophys Acta 1980; $603: 171-7$.

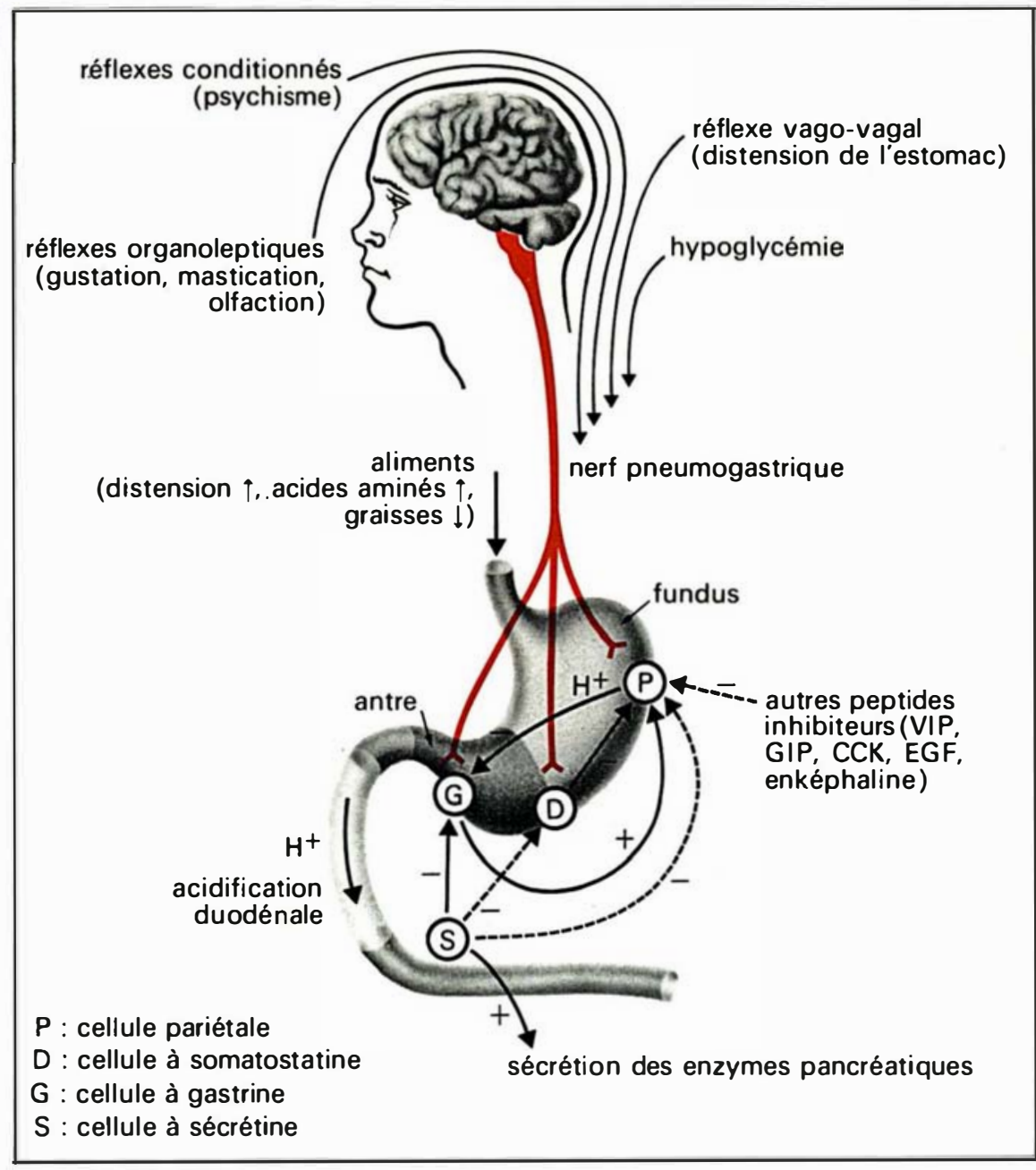

Figure 2. Régulation physiologique de la sécrétion acide gastrique.

La commande nerveuse de la sécrétion acide gastrique utilise l'innervation intrinsèque de la paroi gastrique qui est sensible à la distension provoquée par la présence des aliments. Le nerf pneumogastrique véhicule l'information d'un certain nombre de réflexes liés à la prise alimentaire et transmet aussi le signal déclenché, au niveau des noyaux bulbaires, par l'hypoglycémie caractérisant l'état de jeûne. A côté de l'acétylcholine interviennent aussi certains neuropeptides comme le VIP (vasoactive intestinal peptide) et le GRP (gastrin-releasing peptide) sécrété par les terminaisons nerveuses et agissant soit directement soit indirectement sur la cellule pariétale $(P)$. La commande hormonale proprement dite fait en outre intervenir des peptides produits par les cellules endocrines de l'estomac et de l'intestin. Au niveau de l'estomac deux types de cellules jouent un rôle particulièrement important : la cellule $G$ (à gastrine) localisée dans l'antre gastrique et la cellule $D$ (à somatostatine) localisée à la fois dans l'antre et dans le fundus. La cellule $G$ stimule la cellule pariétale. Elle-même stimulée par les acides aminés alimentaires et par les fïbres vagales à GRP, elle est en revanche inhibée par les fibres nerveuses cholinergiques et par l'acidification de la lumière antrale résultant de la sécrétion de $\mathrm{H}^{+}$(ce qui réalise un effet de rétroaction négative). La cellule $D$ exerce un contrôle inhibiteur sur la cellule $G$ et sur la cellule pariétale. Ce contrôle peut s'exercer par une sécrétion de somatostatine à proximité immédiate de ces cellules (voie paracrine) ou après transport de l'hormone par le sang (voie endocrine). D'autres peptides d'origine extra-gastrique dont le VIP, le GIP (gastric inhibitory peptide), la CCK (cholécystokinine), l'EGF (epidermal growth factor) les enképhalines et, peut être, la sécrétine $(S)$, exercent également un contrôle négatif sur la cellule pariétale.

Le signe + indique une stimulation, le signe -, une inhibition. Trait plein : mécanisme démontré. Trait pointillé : mécanisme probable. 
encore été caractérisé biochimiquement. Ce récepteur pourrait être situé à la fois sur la cellule pariétale et sur d'autres cellules faisant office de relai dans la régulation physiologique de la sécrétion acide [8-9]. L'hypothèse a été proposée selon laquelle un récepteur de ce type pourrait commander la libération d'histamine au niveau d'une cellule cible, non encore identifiée. Cette hypothèse privilégiant le rôle de l'histamine comme médiateur final de la sécrétion acide expliquerait l'efficacité remarquable des antagonistes des récepteurs $\mathrm{H}_{2}$ pour inhiber cette sécrétion. Cependant, un autre schéma reste possible dans lequel les trois récepteurs, histaminique, chholinergique et gastrinique, interagiraient "en parallèle " sur la cellule pariétale. Une telle interaction peut se concevoir au niveau même des récepteurs (par des mécanismes allostériques) aussi bien qu'au délà de ceux-ci par l'intermédiaire des sous-unités de régulation, des seconds messagers, ou des protéines kinases. Des récepteurs spécifiques de plusieurs peptides inhibiteurs (somatostatine, gastric inhibitory peptide ou GIP, epidermal growth factor ou EGF) ont été également décrits sur la cellule pariétale. Cependant, l'implication physiologique de ces récepteurs dans la régulation de la sécrétion acide n'est pas encore prouvée. On ignore tout en outre de leur mécanisme d'action, sauf en ce qui concerne le récepteur de la somatostatine : ce récepteur stimulerait la sous-unité inhibitrice de l'adénylate cyclase [10] et antagoniserait

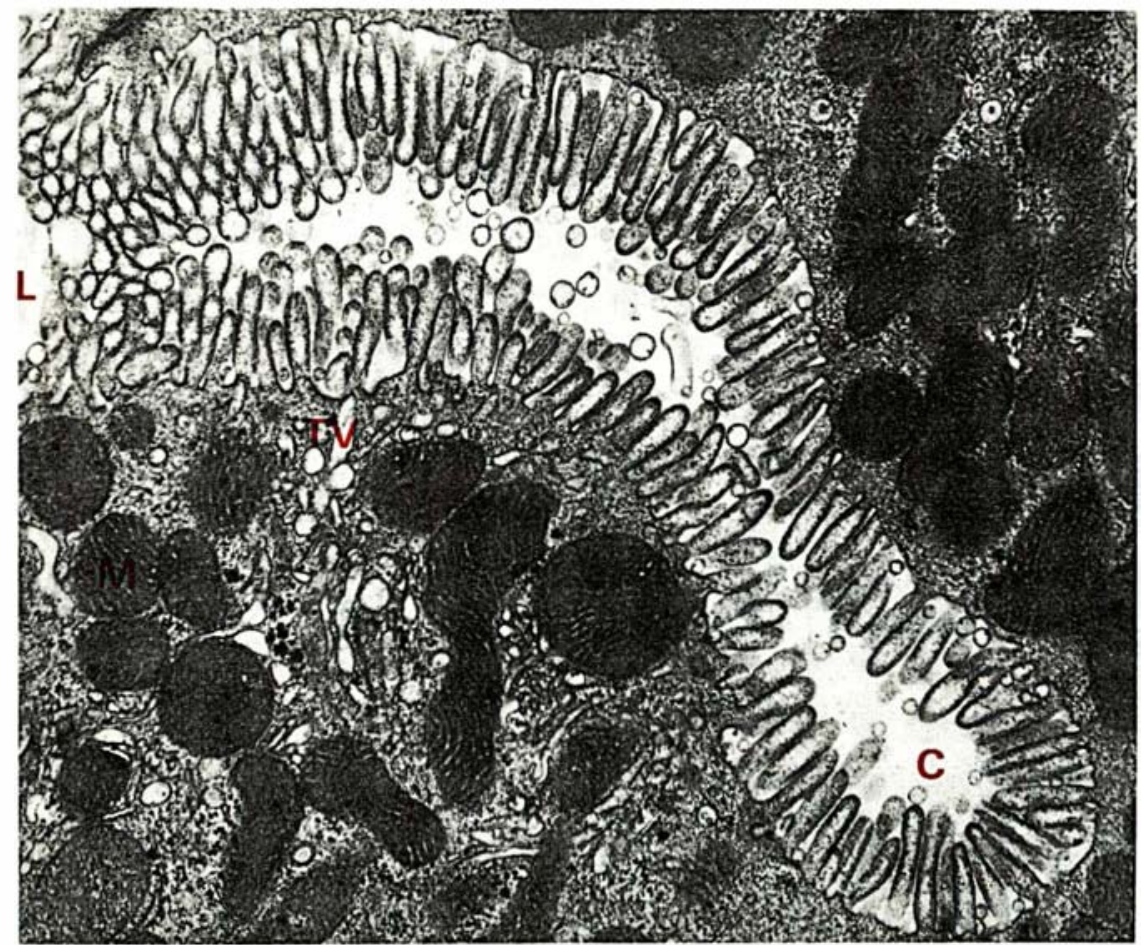

Figure 3. La cellule pariétale vue de près.

Microphotographie de la région apicale d'une cellule pariétale humaine montrant de nombreuses mitochondries (M), quelques tubulovésicules (TV) et une coupe transversale du canalicule intracellulaire $(C)$ aboutissant à la lumière glandulaire (L). Les mitochondries sont nécessaires à la cellule pariétale pour synthétiser l'ATP utilisé par le mécanisme sécrétant $\mathrm{HCl}$ (voir texte). Le canalicule intracellulaire bordé de ses nombreuses microvillosités représente la membrane sécrétoire proprement dite. Il correspond ici à un état d'activité moyen. Au cours de la stimulation, les tubulovésicules fusionnent avec la membrane du canalicule ce qui agrandit considérablement sa surface et permet une sécrétion beaucoup plus importante. aussi l'action des protéines kinases en activant un système de phosphoprotéines phosphatases [11-12].

Un travail de concentration. Compte tenu de son débit important et de sa concentration très élevée en ions $\mathrm{H}^{+}$, la sécrétion acide gastrique représente un mécanisme de transport peu commun. La concentration du proton dans le suc gastrique peut atteindre $150 \mathrm{mEq} / \mathrm{l}$ $(\mathrm{pH} \circ, 8)$ ce qui, comparé à la concentration du proton dans le sang, soit $0,00005 \mathrm{mEq} / 1$ (pH 7,4), représente un rapport de $10^{6,4}$ à 1 . Le même calcul ne s'applique pas à l'ion $\mathrm{Cl}^{-}$puisque la concentration de cet ion dans le suc gastrique (150 mM) est voisine de la concentration sanguine (120 mM). Cependant, le transport de $\mathrm{Cl}^{-}$dans la lumière gastrique s'effectue contre un potentiel électrique défavorable car il existe une différence de plusieurs dizaines de millivolts entre la face séreuse et la face muqueuse (négative) de l'épithélium. Au total, la sécrétion de $\mathrm{HCl}$ doit donc surmonter un important gradient électrochimique. L'énergie nécessaire à cette sécrétion peut être théoriquement estimée à 9460 calories $\left(8400 \mathrm{cal}\right.$ pour l'ion $\mathrm{H}^{+}$et 1060 pour l'ion $\mathrm{Cl}^{-}$). Elle est du même ordre de grandeur que l'énergie libre de l'hydrolyse d'une mole d'ATP à $37^{\circ} \mathrm{C}$ dans l'environnement intracellulaire $(12000$ à 13000 cal). Compte tenu de ces valeurs, la cellule pariétale apparaît comme l'une des cellules les plus "énergétiques" de tout l'organisme. Cette cellule est en eff et particulièrement riche en mitochondries $(40 \%$ du volume cytoplasmique), ce qui lui permet de synthétiser de grandes quantités d'ATP ( figure 3).

Une autre caractéristique explique l'importance quantitative de la sécrétion acide : la membrane apicale de la cellule pariétale est répartie en deux compartiments pouvant s'interchanger, un compartiment de réserve constitué de "tubulovésicules " intracytoplasmique et un compartiment actif, constitué d'une ramification de canalicules bordés de nombreuses microvillosités, pénétrant profondément dans la cellule. La stimulation de la sécrétion acide provoque la migration des 


\section{REFERENCES}

16. Reggio H, Bainton D, Harms E, Coudrier E, Louvard D. Antibodies against lysosomal membranes reveal a 100,000-mol-wt protein that cross-reacts with purified $\mathrm{H}^{+}, \mathrm{K}^{+}$-ATPase of gastric mucosa. I Cell Biol 1984; 99 : 1511-26.

17. Pace CS, Sachs G. Glucose-induced proton upme in secretory granules of cells in monolayer culture. Am 7 Physiol 1982; 242 : C382-7.

18. Scherman D, Nordmann J, Henry JP. Existence of an adenosine $5^{\prime}$-triphosphate dependent proton translocase in bovine neurosecretory granule membrane. Biochemistry 1982; $21: 687-94$.

19. Johnson RG, Beers MF, Scarpa A. $\mathrm{H}^{+}$ATPase of chromaffin granules. 7 Biol Chem 1982 ; 257 : 10701-7.

20. Stone DK, Xie XS, Racker E. An ATPdriven proton pump in clathrin-coated vesicles. 7 Biol Chem 1983; $158: 4059-62$.

21. Smolka A, Helander HF, Sachs G. Monoclonal antibodies against gastric $\mathrm{H}^{+}, \mathrm{K}^{+}$-ATPase. Am 7 Physiol 1983; 245 : G589-96.

22. Sachs G, Chang HH, Rabon E, Schackman R, Lewin MJM, Saccomani G. A nonelectrogenic $\mathrm{H}^{+}$pump in plasma membrane of hog stomach. $\mathcal{F}$ Biol Chem 1976; 251 : 7690-8.

23. Faller L, Jackson R, Malinowska D et al. Mechanistic aspects of gastric $\left(\mathrm{H}^{+}+\mathrm{K}^{+}\right)-\mathrm{ATPase}$. Ann NY Ac ad Sci 1982 ; 156-63.

24. Lewin MJM. Molecular mechanisms of gastric acid secretion. In: Desnuelle $P$, Nören $O$, Sjöström H, ed. Molecular and Cellular Biology of Digestion. Amsterdam: Elsevier-North Holland, 1985 (in press)

25. Fellenius E, Elander B, Wallmark B, Helander $F$, Berglindh $T$. Inhibition of acid secretion in isolated gastric glands by substituted benzimidazoles. Am 3 Physiol 1982; 243 : G505-10.

26. Lewin MJM. Oméprazole: un nouveau mécanisme d'action et une efficacité sans précédent dans le traitement de l'hypersécrétion acide gastrique. Gastroenterol Clin Biol 1984; 8 : 93942.

27. Soumarmon A, Grelac F, Lewin MJM. Solubilization of active $\left(\mathrm{H}^{+}, \mathrm{K}^{+}\right)$-ATPase from gastric membrane. Biochim Biophys Acta 1983; $732: 579-85$

28. Rabon E, Gunther RD, Soumarmon A, Bassilian S, Lewin MJM, Sachs G. Solubilization and reconstitution of the gastric $\mathrm{H}, \mathrm{K}-\mathrm{ATPase} .7$ Biol Chem 1985; 259 (in press).

29. Soumarmon A, Abastado M, Bonfils S, Lewin $\mathrm{MJM} . \mathrm{Cl}^{-}$transport in gastric microso mes. An ATP-dependent influx sensitive to membrane potential and to protein kinase inhibitor. I B iol Chem 1980; $255: 11682-7$.

30. Wolosin JM, Forte JG. Stimulation of oxyntic cell triggers $\mathrm{K}^{+}$and $\mathrm{Cl}^{-}$conductances in apical $\left(\mathrm{H}^{+}, \mathrm{K}^{+}\right)$-ATPase membrane. Am $\mathcal{F}$ Physiol tubulovésicules vers le pôle apical et leur fusion avec la membrane des canalicules. Il en résulte une augmentation considérable de la surface sécrétoire. Ainsi, si l'on tient compte du nombre de cryptes glandulaires par centimètre carré de surface de muqueuse fundique et du facteur de multiplication apporté par la présence des canalicules intracellulaires, la surface sécrétoire réelle correspondant aux quelques 900 millions de cellules pariétales contenues dans l'estomac, représente une aire de plusieurs dizaines de mètres carrés [13].

\section{La $\left(H^{+}, K^{+}\right)$-ATPase}

Des expériences récentes réalisées in vitro sur des glandes ou des cellules gastriques isolées et rendues perméables à l'ATP par un détergent doux, ont permis de montrer que ce nucléotide était nécessaire et suffisant pour la mise en route et l'entretien de la sécrétion de l'ion $\mathrm{H}^{+}$. Ce rôle essentiel de l'ATP était suspecté depuis longtemps et avait orienté les recherches sur le mécanisme du transport de $\mathrm{H}^{+}$ vers la caractérisation d'une ATPase membranaire spécifique.
Cette ATPase a été effectivement découverte en 1973 [14]. Cette nouvelle enzyme se distingue des autres ATPases déjà connues par plusieurs critères. Comme la $\left(\mathrm{Na}^{+}, \mathrm{K}^{+}\right)$ATPase des membranes plasmiques, elle passe, au cours de son cycle catalytique, par une forme phosphorylée à laquelle est associée une activité phosphatasique. Cette propriété la distingue de l'ATPase à protons mitochondriale. $\mathrm{Ce}$ pendant, contrairement à la $\left(\mathrm{Na}^{+}\right.$, $\mathrm{K}^{+}$)-ATPase et à la $\mathrm{Ca}^{2+}$ ATPase du réticulum sarcoplasmique, ni l'ion $\mathrm{Na}^{+}$, ni l'ion $\mathrm{Ca}^{2+}$ ne sont nécessaires à son fonctionnement. De plus, elle est insensible à l'ouabaïne, inhibiteur spécifique de la $\left(\mathrm{Na}^{+}, \mathrm{K}^{+}\right)$ATPase.

L'ATPase gastrique est stimulée par les cations monovalents avec une nette préférence pour l'ion $\mathrm{K}^{+}$, ce qui explique son nom: $\left(\mathrm{H}^{+}\right.$, $\mathrm{K}^{+}$)-ATPase. Elle est spécifique de la cellule pariétale mais pourrait cependant appartenir à la même famille que d'autres ATPases à protons présentes sur la membrane de divers organites à contenu acide : lysosomes, granules sécrétoires, vésicules d'endocytose $\lceil 1$ 5-20]. La

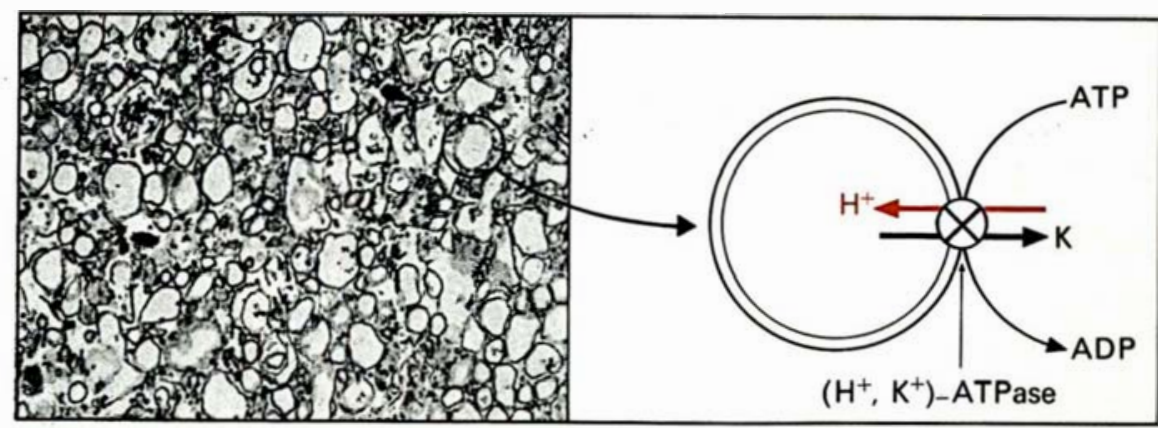

Figure 4. La sécrétion acide réduite à sa plus simple expression.

La microphotographie de gauche montre des vésicules de membranes cellulaires préparées à partir d'estomac de porc utilisées comme modèle in vitro pour l'étude du transport de $H^{+}$par I ATPase gastrique. Elles représentent les tubulovésicules de la cellule pariétale et aussi des fragments de la membrane canaliculaire refermés sur eux-mêmes au cours de la préparation. Incubées en présence d'ATP (et de $\mathrm{Mg}^{2+}$ ) ces vésicules manifestent la capacité de transporter des ions $H^{+}$, de lextérieur vers lintérieur en échange d'ions transportés dans lautre sens. Cette propriété est due à lactivation par lATP de la $\left(H^{+}, K^{+}\right)$-ATPase selon le mécanisme schématisé à droite. Compte tenu de l'orientation de la membrane (la face externe de la vésicule correspond à la face cytosolique) ce transport donne une image inversée de la sécrétion des protons dans la lumière gastrique. 
localisation de la $\left(\mathrm{H}^{+}, \mathrm{K}^{+}\right)$-ATPase dans la cellule pariétale a été précisée par immunocytochimie à l'aide d'anticorps monoclonaux [21]. Comme on pouvait le prédire d'un effecteur de la sécrétion acide, elle est spécifiquement située sur les membranes des tubulovésicules et des canalicules intracellulaires.

La mise en évidence et la caractérisation d'un transport de $\mathrm{H}^{+}$associé au cycle catalytique de l'ATPase gastrique ont été effectuées sur des fractions purifiées de membranes apicales de cellules pariétales [22] Ces fractions se présentent sous la forme de vésicules de quelques dizièmes de micromètre de diamètre (figure 4) qui correspondent soit aux tubulovésicules natives de la cellule pariétale, soit à des fragments de membrane canaliculaire qui se sont refermés sur eux-mêmes au cours de la préparation. Dans ce dernier cas, plus de la moitié des vésicules ainsi formées sont éversées (inside-out), la face de la membrane qui était à l'origine tournée vers le cytoplasme se retrouvant tournée vers le milieu extérieur. Il est donc possible d'utiliser de telles tubulovésicules et vésicules éversées pour tester "en miroir" l'activité de transport de la $\left(\mathrm{H}^{+}, \mathrm{K}^{+}\right)$-ATPase, en mesurant l'accumulation de protons dans ces vésicules en réponse à une addition d'ATP dans le milieu. Cette accumulation est mesurée soit directement par pH-métrie (on mesure en fait l'alcalinisation du milieu extravésiculaire), soit en utilisant comme sondes des molécules portant un chromophore ou un fluorophore sensible au $\mathrm{pH}$ intravésiculaire ou au gradient transmembranaire de protons. Il a été ainsi montré que la $\left(\mathrm{H}^{+}, \mathrm{K}^{+}\right)$-ATPase catalysait en présence d'ATP (et de magnésium) l'échange stæchiométrique d'un ion $\mathrm{H}^{+}$transporté de la phase cytosolique vers la face luminale de la membrane contre un ion $\mathrm{K}^{+}$ transporté dans l'autre sens ( $f \hat{i}$ gure 4). Le cycle de réactions réalisant cet échange et aboutissant à l'hydrolyse de l'ATP ferait intervenir trois états conformationnels successifs ( figure 5) [23-24]. Ce cycle réactionnel impliquerait un ou plusieurs groupe(s) SH nécessaire(s) à la fixation et à l'hydrolyse de $m / s n^{0} 5$ septembre 85

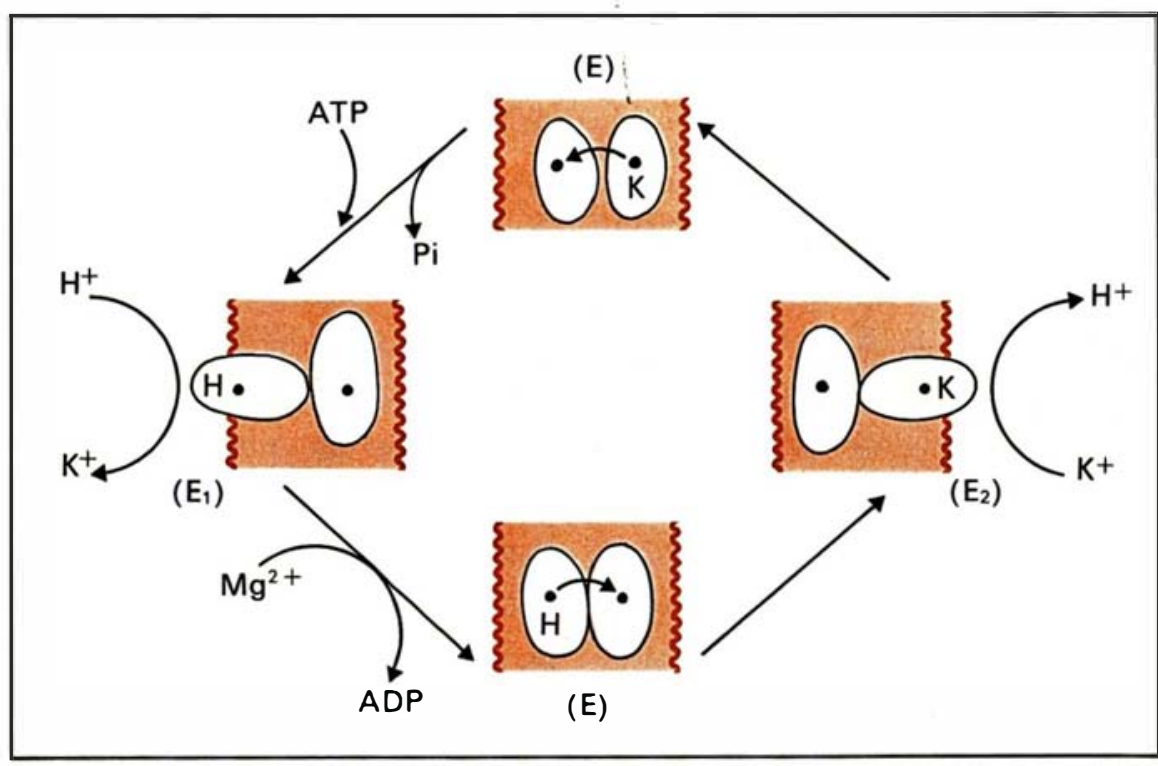

Figure 5. Comment "fonctionne "la $\left(\mathrm{H}^{+}, \mathrm{K}\right)$-ATPase?

Ce modèle théorique illustre le fonctionnement probable de la $\left(\mathrm{H}^{+}, \mathrm{K}^{+}\right)$-ATPase. $L$ 'enzyme est ici représentée sous forme dimérique. Dans un premier état [ $\left.E_{2}\right]$ capable de lier l'ATP, l'enzyme émerge à la face cytosolique de la membrane et présente une grande affinité pour l'ion $H^{+}$et une faible affinité pour l'ion $K^{+}$. Dans cet état, cette forme de l'enzyme fixe un ion $\mathrm{H}^{+}$. En présence de magnésium $\left(\mathrm{Mg}^{2+}\right)$, l'ATP est hydrolysé et l'enzyme est convertie dans un état correspondant à la forme phosphorylée [E]. Dans cet état le proton change vraisemblablement de site (flèche). L'enzyme passe ensuite au stade [E2], symétrique de l'état [ $\left.E_{2}\right]$, dans lequel il émerge à la face luminale de la membrane et présente cette fois une grande affinité pour l'ion $K^{+}$et une faible affinité pour l'ion $H^{+}$. Le proton est donc déplacé par $K^{+}$et se retrouve dans le compartiment luminal. L'enzyme repasse ensuite dans l'état [E] dans lequel le potassium change probablement de site et, après déphosphorylation, retourne finalement à l'état $\left[E_{\imath}\right]$ de départ. L'enzyme fixe alors une nouvelle molécule d'ATP et l'ion $K^{+}$est remplacé par un nouveau proton. Au total le cycle réalise l'échange stochiométrique de l'ion $\mathrm{H}^{+}$contre l'ion $\mathrm{K}^{+}$à travers la membrane.

l'ATP. Les inhibiteurs chimiques de tels groupes sont donc susceptibles de bloquer l'activité de la $\left(\mathrm{H}^{+}\right.$, $\mathrm{K}^{+}$)-ATPase. C'est le cas d'une nouvelle série de molécules dérivées du benzimidazole et dont le chef de file est l'" oméprazole" [25]. Ces dérivés ont de plus l'avantage d'être des bases faibles, ce qui les conduit à s'accumuler sélectivement dans les compartiments acides de la cellule pariétale (tubulovésicules et canalicules). Cette double propriété fait de tels "anti-ATPase" des inhibiteurs remarquablement efficaces de la sécrétion acide gastrique [26]. La $\left(\mathrm{H}^{+}, \mathrm{K}^{+}\right)$-ATPase serait constituée de plusieurs sous-unités de poids moléculaire voisin de 100000 daltons. Il a été récemment possible de l'extraire des membranes gastriques sous forme soluble et de la réincorporer dans des vésicules artificielles (liposomes) préparées à partir d'un mélange de phospholipides [27-28]. L'étude de l'enzyme ainsi reconstituée devrait permettre de mieux préciser sa structure et son fonctionnement moléculaire. Une autre approche également en cours vise à caractériser son codage génétique.

$$
\mathrm{H}^{+}+\mathrm{Cl}^{-}=\mathrm{HCl}
$$

Le transport de l'ion $\mathrm{Cl}^{-}$accompagnant l'ion $\mathrm{H}^{+}$dans la lumière gastrique est moins bien connu. Certaines observations suggèrent que ce transport s'effectuerait à travers un canal spécifique situé également sur la membrane apicale de la 
cellule pariétale et dont l'ouverture serait commandée par une protéine kinase dépendante de l'AMP cyclique [29]. Il s'agirait d'un système à voie unique (uniport) dont le fonctionnement ne serait donc pas électriquement neutre, chaque $\mathrm{Cl}^{-}$ transporté ajoutant une charge négative sur la face luminale de la membrane. Contrairement à la $\left(\mathrm{H}^{+}, \mathrm{K}^{+}\right)$-ATPase qui représente pour le transport de $\mathrm{H}^{+}$un mécanisme énergétique capable de créer

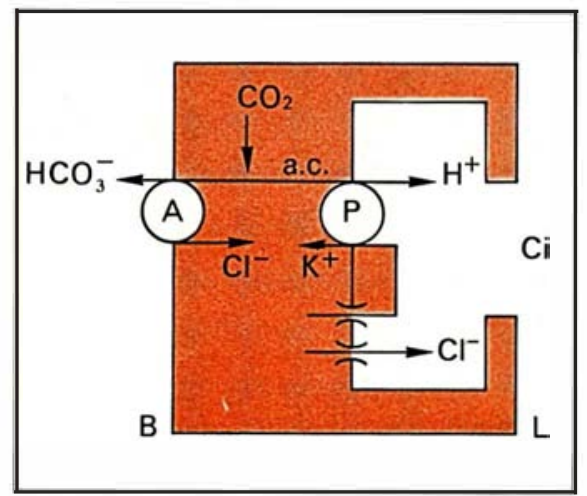

Figure 6. Un schéma d'ensemble. Schéma simplifié illustrant la sécrétion de $\mathrm{HCl}$ par la cellule pariétale gastrique. Cette sécrétion est assurée par deux mécanismes associés sur la membrane du canalicule intracellulaire $(C i)$ : d'une part la $\left(H^{+}, K^{+}\right)$-ATPase $(P)$, d'autre part un canal $\mathrm{Cl}^{-}$. L'ouverture de ce canal est vraisemblablement commandée par l'AMP cyclique en réponse à la stimulation des récepteurs situés sur la membrane basale (B) non représentés ici. Lorsque la cellule est stimulée, le transport d'un ion $\mathrm{Cl}^{-}$entraîne, par couplage électrique, celui d'un ion $K^{+}$. Ce dernier retourne dans la cellule en échange d'un ion $\mathrm{H}^{+}$ au niveau de la $\left(H^{+}, K^{+}\right)$-ATPase. L'ion $\mathrm{OH}^{-}$restant dans la cellule est évacué, après une carbonatation catalysée par l'anhydrase carbonique (a.c.), à l'aide d'un échangeur $\mathrm{HCO}_{3}^{-} / \mathrm{Cl}^{-}$situé au niveau de la membrane basale $(A)$. Sur cette membrane une $\left(\mathrm{Na}^{+}\right.$, $K^{+}$)-ATPase (non représentée) assure l'entrée de $\mathrm{K}^{+}$et active l'entrée de $\mathrm{Cl}^{-}$ par l'intermédiaire d'un système couplé au gradient de $\mathrm{Na}^{+}$. un important gradient de concentration, le canal $\mathrm{Cl}^{-}$de la membrane luminale n'est pas un transporteur actif. Un tel système n'apparaît d'ailleurs pas nécessaire puisque la captation de l'ion $\mathrm{Cl}^{-}$au niveau de la membrane basolatérale est elle-même activée par le gradient de $\mathrm{Na}^{+}$créé par la $\left(\mathrm{Na}^{+}\right.$, $\mathrm{K}^{+}$)-ATPase. Ce même gradient de $\mathrm{Na}^{+}$est sans doute aussi responsable de la sécrétion de $\mathrm{NaCl}$ accompagnant la sécrétion acide lorsque celle-ci est peu activée (entre les repas).

Le transport "électrogénique " du $\mathrm{Cl}^{-}$serait à l'origine de la différence de potentiel transépithéliale déjà évoquée. Cette différence de potentiel qui peut atteindre $60 \mathrm{mV}$ à l'état basal diminue jusqu'à $-30 \mathrm{mV}$ lorsque la sécrétion acide se met en route. Cette diminution est due à la baisse de résistance transépithéliale résultant de l'augmentation de la surface sécrétoire et à un effet de couplage électrique entre la sécrétion de $\mathrm{H}^{+}$et celle de $\mathrm{Cl}^{-}$. Ce couplage ne met pas directement en cause l'ion $\mathrm{H}^{+}$dont le transport, nous l'avons vu, est réalisé par un échange stœchiométrique électriquement neutre. Il correspond vraisemblablement à l'ouverture d'un autre canal permettant le passage de l'ion $\mathrm{K}^{+}$, du cytosol vers la lumière gastrique [30]. Puisque $\mathrm{K}^{+}$est nécessaire au fonctionnement de la $\left(\mathrm{H}^{+}, \mathrm{K}^{+}\right)$-ATPase, le transport de cet ion dans le compartiment luminal est en eff et indispensable.

Ainsi, la sécrétion de $\mathrm{HCl}$ par la cellule pariétale gastrique serait le résultat de deux mécanismes distincts, mais fonctionnellement couplés par un cycle $\mathrm{K}^{+}$(figure 6 ). Le proton transporté dans cette sécrétion provient de l'eau cellulaire d'où il est extrait par l'ATPase qui laisse en place l'ion $\mathrm{OH}^{-}$. Cet ion est évacué au pôle basal de la cellule, sous forme de $\mathrm{HCO}_{3}^{-}$, après une carbonatation catalysée par l'anhydrase carbonique, la sortie de l'ion bicarbonate étant couplée à l'entrée d'un ion $\mathrm{Cl}^{-}$. La sécrétion de $\mathrm{HCl}$ étant approximativement isotonique, l'eau qui l'accompagne est vraisemblablement drainée vers la lumière gastrique par simple effet osmotique

\section{Summary}

Our stomach secretes each day up to 2-3 liters of a highly concentrated hydrochloric acid solution ( $\left.\mathrm{pH}_{1}\right)$. This "acid" secretion is achieved by the so called "parietal" cells located in the epithelial glandular tubes of the upper part of the stomach. These cells possess a large number of mitochondria producing the energy required by the $\mathrm{H}^{+}$ pump and an intracellular canaliculus whose size can expand to form a very large secretory surface. Physiological regulation of parietal ccll activity is a complex process integrating both local and central reflexes transmitted by the pneumogastric nerve together with hormonal stimuli. The neuromediator, acetylcholine, the peptide hormone, gastrin, and the biogene amine, histamine, are the three main stimulants of acid secretion. They act on the parietal cells through specific receptors which activate the secreting machinery by the way of second messengers such as cyclic AMP and calcium. The effector system of $\mathrm{H}^{+}$secretion is the $\left(\mathrm{H}^{+}, \mathrm{K}^{+}\right)$-A TPase. This recently discovered enzyme is specific to the parietal cells. The enzyme is suggested to operate a neutral exchange transporting one $\mathrm{H}^{+}$ion from the cytosolic to the luminal side of the parietal cell membrane against one $\mathrm{K}^{+}$ ion entering the cell from the gastric lumen. It is functionally associated with a $\mathrm{Cl}^{-}$transport controlled by cyclic AMP. Upon stimulation, a $\mathrm{K}^{+}$channel also opens on the secretory membrane which allows $\mathrm{K}^{+}$ ions to accompany $\mathrm{Cl}^{-}$ions into the lumen. These $\mathrm{K}^{+}$ions are then recycled into the cell by the $\left(\mathrm{H}^{+}, \mathrm{K}^{+}\right)$-ATPase.

\section{TIRES A PART}

MJM Lewin : Inserm U10, hôpital Bichat, 170, boulevard Ney, 75877 Paris cedex 18 